\title{
Creative
}

\section{Realization of Maxwell's Hypothesis: A Heat-Electric Conversion in Contradiction to the Kelvin Statement}

\author{
Xinyong $\mathrm{Fu}^{*,+}$ and $\mathrm{Zitao} \mathrm{Fu} \ddagger$ \\ Shanghai Jiao Tong University, 800 Dongchuan RD., Minhang District, Shanghai, China \\ * Correspondence: xyfu@sjtu.edu.cn \\ $+\quad$ This author retired in1998 (at 60 ). \\ $\ddagger \quad$ This author is now a manager in a company in Shanghai.
}

\begin{abstract}
In a vacuum tube, two identical and parallel Ag-O-Cs surfaces, with a work function of approximately $0.8 \mathrm{eV}$, ceaselessly emit thermal electrons at room temperature. The thermal electrons are so controlled by a static uniform magnetic field that they can fly only from one Ag-O-Cs surface to the other, resulting in a potential difference and an electric current, and transferring a power to a resistance outside the tube. The ambient air is a single heat reservoir in the experiment, and all the heat extracted by the tube from the air is converted into electric energy without producing other effect. The authors maintain that the experiment is in contradiction to the Kelvin statement of the second law of thermodynamics. We have a video on you tube showing the main measuring process of the experiment: https://www.youtube.com/watch?v=PyrtC2nQ UU.
\end{abstract}

Keywords: Maxwell's demon; entropy decreasing; energy regeneration; energy circulation

\section{Fundamental Concept}

In a vacuum tube there is a quartz plate whose upper surface is coated with two identical and parallel thermal electron emitters, A and B, as shown in Fig.1. A gap between A and B insulates them from each other. The whole tube is immersed in a single-temperature heat reservoir whose temperature is such that A and B ceaselessly emit thermal electrons.

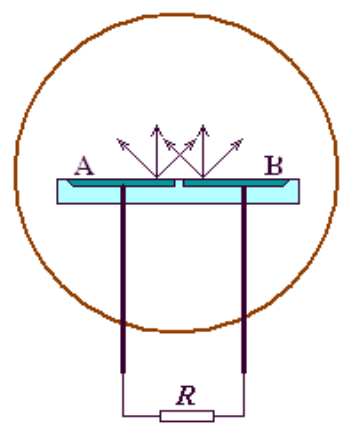

Fig.1 Two identical thermal electron emitters, $\mathrm{A}$ and $\mathrm{B}$, are set parallel in a vacuum tube.

Fig.2 (a) illustrates the motion of the thermal electrons emitted from two points located symmetrically on A and B while no magnetic field is applied to the tube. Some electrons emitted by A can travel across the gap and fall on B, and simultaneously an 


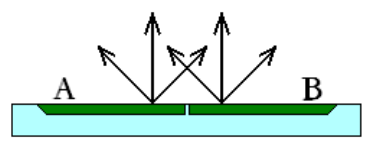

(a) $B=0$

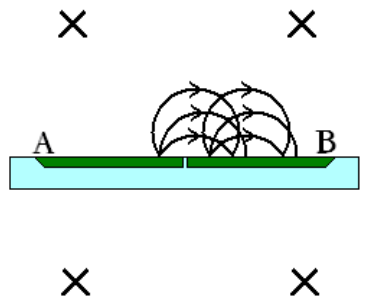

(b) $B>0$

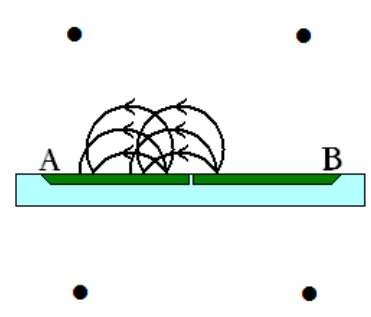

(c) $B<0$

Fig. 2 Thermal electrons' motion with or without a magnetic field.

approximately equal number of electrons emitted by B can also travel across the gap and fall on A. The two tendencies cancel, resulting in no net charge (whether positive or negative) on A or B.

Now, if a static uniform magnetic field is applied to the tube in the direction parallel to the gap, the paths of electrons will change into circles with different radii, swifter electrons flying along larger circles and slower ones along smaller circles. As shown in Fig.2 (b), many of the electrons emitted by A can travel across the gap and fall on B, but it is now impossible for electrons emitted by $B$ to travel across the gap and fall on A. Such a net transition of electrons from A to B will rapidly result in a charge distribution, with A charged positively and B charged negatively. A potential difference between A and B is established, resulting in a direct current (which may be referred to as Maxwell's current) and an electric power both transferred to a resistor or a reversible battery outside the tube ${ }^{[1]}{ }^{[2]}$. (The above discussion neglects the effect of thermal electrons' collisions with the glass tube wall, otherwise, due to the collisions, some of the electrons emitted by B may also fly across the gap to fall on A. Nevertheless, mostly, the flow of electrons from A to B prevails over the opposite flow.)

Reverse the direction of the magnetic field, as shown in Fig.2 (c), the direction of the electrons' motion and the output current reverse, too.

There is a problem here: where does the electric power originate?

It is the heat extracted by the electron tube from the heat reservoir (in which the tube is immersed) that provides the power. We explain this heat-electric conversion process as follows.

As A is charged positively and B charged negatively, a static electric field between them (especially in the region above the gap between $A$ and $B$ ) emerges immediately. The direction of the electric field is to resist the succeeding thermal electrons' flight from A to B.

Let's investigate the flight process of the electrons from A to B. As an example, at the upper part of fig.3, we see an electron with a velocity $v$ is flying rightward, and the force exerted on it by the static electric field $F$ is left-ward, so the electron will be decelerated by the force. Nevertheless, a certain part of the electrons emitted by A (chiefly the faster ones), relying on their kinetic energy, can overcome the resistance of the static electric field and travel across the gap to fall on $B$. On arriving at $B$, each electron obtains an amount of electric potential energy in exchange for an equal amount

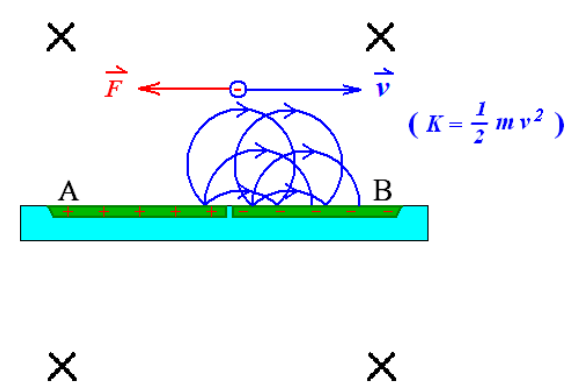

Fig.3, $v$, rightward, is the speed of an electron flying above the gap between A and B. F, leftward, is the force exerted by the static electric field on the electron, resisting its flight from A to $B$. The kinetic energy of the faster electrons enables them to overcome the resistance. 
of the electron's kinetic energy. Thus these electrons "cool down". Consequently the two emitters and the $\mathrm{n}$ the whole ele tron tube also cool $\mathrm{d}$ own (maybe ve $\mathrm{y}$ slightly.) As ele tric e rergy is continuously transferred to the outside resistance, the tube is continuously losing its internal energy and cools down, which is compensated by the heat from the surrounding heat reservoir.

In the above process, the electron tube extracts heat from a single heat reservoir and all the heat is converted into electric energy without producing other effect. We maintain that the process is in contradiction to the Kelvin statement of the second law of thermodynamics.

As is well known, in 1871, to challenge the absoluteness of the second law of thermodynamics, James Clerk Maxwell came up with a famous hypothesis - Maxwell's demon [3] [4]. According to Maxwell, Ehrenburg and some others, this so-called demon may work in either of the two following modes ${ }^{[5]}$.

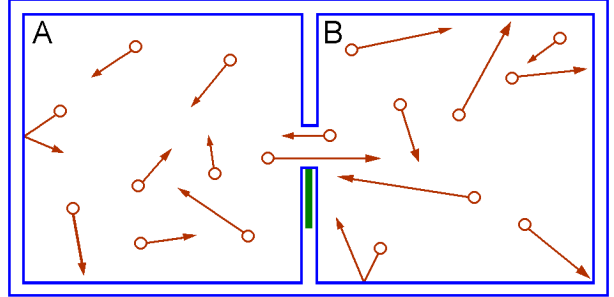

(a) In the first mode, the demon produces an inequality in temperature between $\mathrm{A}$ and $\mathrm{B}$

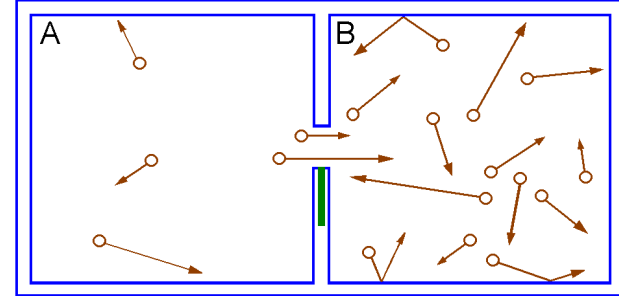

(b) In the second mode, the demon produces an inequality in pressure between $\mathrm{A}$ and $\mathrm{B}$

Fig. 3 Maxwell's demon interferes with the random thermal motion of gas molecules*

In the first mode, as shown in Fig.3 (a), the demon allows only the swifter molecules to pass through a small doorway and move from $A$ to $B$, and the slower ones to pass through the doorway from $\mathrm{B}$ to $\mathrm{A}$, causing eventually a difference in temperature between $\mathrm{A}$ and $\mathrm{B}$.

In the second mode, as shown in Fig.3 (b), the demon only allows the molecules to pass through the doorway from A to B, causing eventually a difference in pressure between A and B.

In our present design, the magnetic field functions as the above mentioned demon, working in the second mode: It allows thermal electrons only to flight from A to B, causing a difference in electric potential between A and B.

The following is an actual experiment we performed recently, showing how thermal electrons in a vacuum tube move in a magnetic field, causing an electric potential, a current, and an output power.

\section{THE ELECTRON TUBE USED}

\section{A. Choice of thermal electron emitters and working temperature}

In principle, any of the thermal electron cathode materials known today may be used for such an experiment. However, in order to accomplish an original and straightforward experiment, we chose Ag-O-Cs cathode material. Ag-O-Cs has the lowest work function among all the known thermal electron materials, about $0.8 \mathrm{eV}$, and is currently optimum in maximizing thermal electron emission at room temperature ${ }^{[6]}$. We adopted this material and let the tube and the entire closed circuit shown in Fig.1 to operate under a uniform room temperature, so as to avoid disturbances arising from the Seebeck effect, etc.

Ag-O-Cs cathodes are in nowadays widely used in photoelectric tubes and photomultipliers, and their emission of thermal electrons is commonly referred to as dark current. Users certainly prefer weak-dark-current Ag-O-Cs cathodes. Manufacturers adjust their technology to produce cathodes with a low dark current, usually in the range $10^{-11}$ to $10^{-14} \mathrm{~A} / \mathrm{cm}^{2}$. In our experiment, on the contrary, we desired to use emitters of strong-dark-current. The authors adjusted the manufacturing technology repeatedly over the past 18 years and succeeded in producing tubes with Ag-O-Cs 
emitters with a dark current in the range $10^{-7}$ to $10^{-10} \mathrm{~A} / \mathrm{cm}^{2} .^{[7]}$

In this experiment, the tube plays the role of an electric power source, and the load of the power source may be a resistor or a reversible battery. In our present tests, we used the input resistor of an electrometer, Keithley 6514, together with (in series) a resistor of $150 \mathrm{M}$ as the load. In such an arrangement, the electrometer might simultaneously be used to measure the output current or output voltage produced by the electron tube. Working at room temperature, the whole closed circuit was readily kept at a rather uniform temperature.

\section{B. The structure of the electron tube}

The electron tube used in the present experiment was an FX12 (actually FX12-51), whose structure is shown in Fig.4. The envelope was of glass. A and B, see Fig. 4 (a), were two identical Ag-O-Cs thermal electron emitters mounted on the tops of two parallel copper bars. Between the two copper bars there was a mica sheet (i.e. the "gap"), keeping A and B mutually insulated. The mica sheet projected out approximately $7 \mathrm{~mm}$ at the middle of the bottom of the two copper bars (out the gap) to

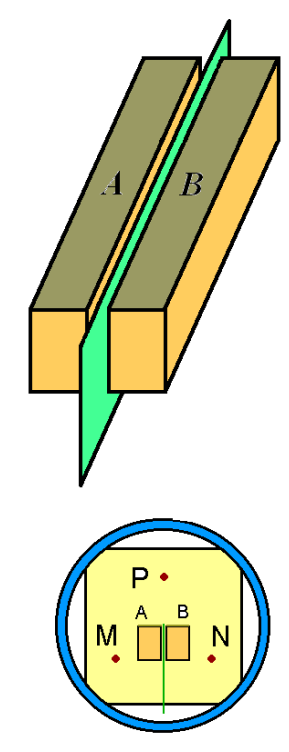

(a) Emitters A and B, a mica sheet, rods $P, M, N$, supports.

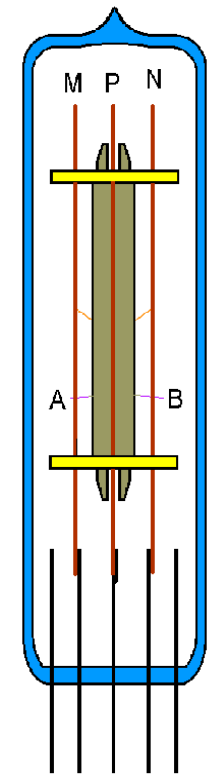

(b) Sketch of the structure of electron tube FX12-51

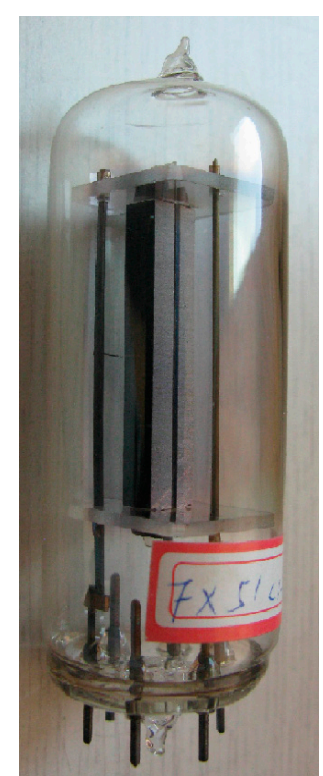

(c) A photograph of FX12-51 (i.e., FX51 (12))

Fig. 4 Electron tube FX12-51

prevent electrons returning from B to A by flying (cycling) beneath the two bars. $\mathrm{M}, \mathrm{N}$ and $\mathrm{P}$ were three molybdenum supporting rods. $\mathrm{M}$ and $\mathrm{N}$ were simultaneously used as electrical leads separately connecting A and B to the load outside the tube. P was $6 \mathrm{~mm}$ above the gap, and was used as a temporary anode in the tube manufacturing process to oxidize the silver films of $A$ and $B$ by oxygen-discharge. After the manufacture of the tube, $\mathrm{P}$ was again used as a temporary anode to measure the dark current of the two emitters to check the quality of the tube. The typical dark current of each emitter was $500 \sim 500,000 \mathrm{pA}$.

Finally, when manufacture the tubes, the leakage resistance between A and B should be at least greater than $100 \mathrm{M} \Omega$. The value of the leakage resistance depends chiefly on the amount of cesium input during the manufacture.

\section{MEASUREMENT OF THE MAGNETIC FIELD AND OUTPUT CURRENT}

\section{a) The magnetic field}

The magnetic field used to deflect the orbits of the thermal electrons was produced by a $150 \mathrm{~mm}$ $\times 100 \mathrm{~mm} \times 25 \mathrm{~mm}$ magnet. Fig. 5 shows the magnetic induction intensity $B$ at point $O$ on the axis of the magnet, a distance $d$ from the magnet. The $B \sim d$ relation was measured in advance with a teslameter, and the results are listed in Table 1. In our experiment of thermo-electric conversion, the 
electron tube was placed at point $\mathrm{O}$, within a shielding box, with the tube axis parallel to the magnetic field.

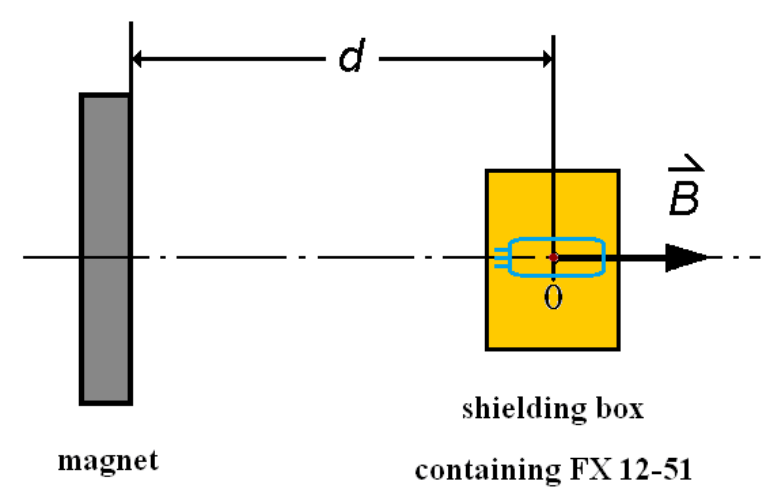

Fig. 5 The magnetic field produced by a magnet used in the experiment Table $1 B \sim d$ relation of the magnet.

\begin{tabular}{|c|c|c|c|c|c|c|c|c|c|c|}
\hline$d(\mathrm{~cm})$ & 70 & 60 & 50 & 40 & 35 & 30 & 25 & 20 & 15 & 10 \\
\hline $\begin{array}{l} \\
\text { (N)(gaus) }\end{array}$ & 0.2 & 0.3 & 1.1 & 2.1 & 2.9 & 4.4 & 7.2 & 13.1 & 25.5 & 59.7 \\
\hline$B_{\downarrow}(\mathrm{S})$ (gaus) & -0.6 & -0.8 & -0.7 & -1.6 & -2.5 & -4.0 & -6.7 & -12.7 & -24.9 & -58.8 \\
\hline $\boldsymbol{B}_{(a b s, \text { mean })}$ & 0.4 & 0.6 & 0.9 & 1.9 & 2.7 & 4.2 & 7.0 & 13 & 25 & 60 \\
\hline
\end{tabular}

b) The output current and voltage

The output current of the electron tube was measured using a Keithley 6514 electrometer with a highest current sensitivity of $1 \times 10^{-16} \mathrm{~A}=0.1 \mathrm{fA}$. A simple diagram of the measuring circuit is shown in Fig. 6.

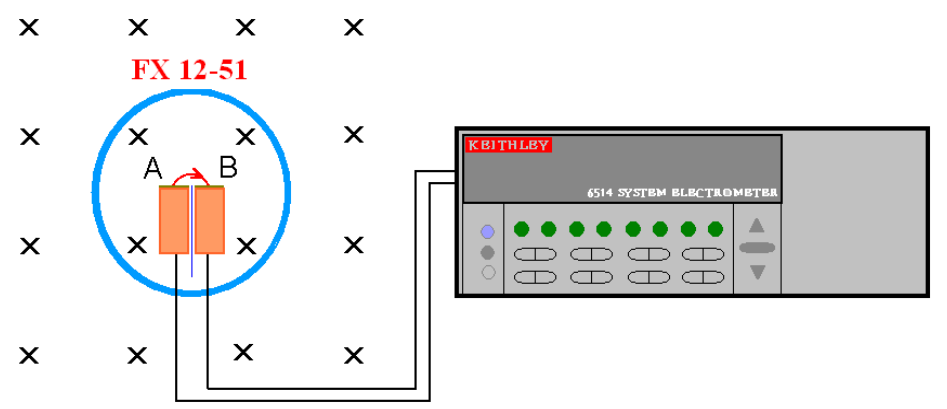

Fig.6 Current measuring circuit.

In addition to the output current, the electrometer with the circuit was also used to measure the output voltage of the electron tube. The highest voltage sensitivity of Keithley 6514 is $1 \times 10^{-5} \mathrm{~V}=$ $0.01 \mathrm{mV}$.

\section{THE EXPERIMENT}

Fig.7 (a) is a photograph of the set up of the experiment. From left to right: a Keithley 6514 electrometer, a copper shielding box (containing electron tube FX12-51), and a magnet. Fig. 7 (b) shows how the electron tube lay within the copper shielding box. The anticipated output current or output voltage caused by static magnetic field was transferred to the electrometer through a special accessory cable. 


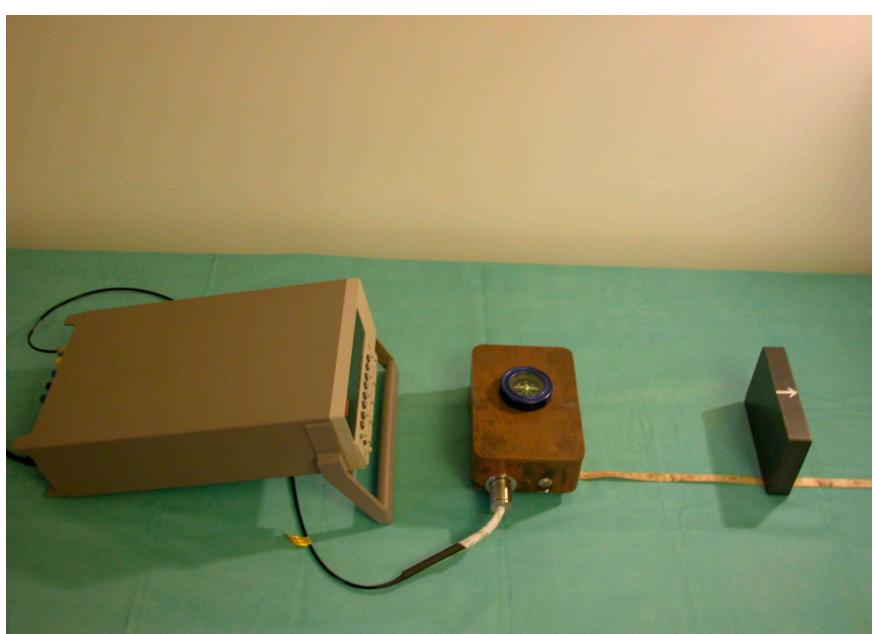

(a)

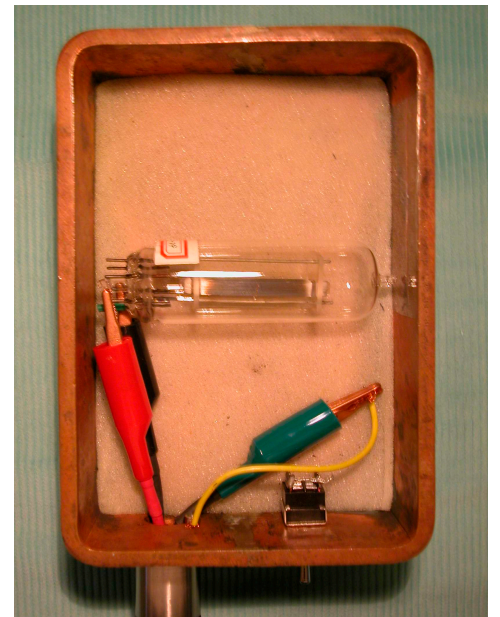

(b)

(a) A Keithley 6514 electrometer, a copper shielding box (containing FX12-51), and a magnet. (b) Position and orientation of electron tube FX12-51 in the copper shielding box.

Fig. 7 Set up of the experiment

First, we chose a room temperature, which should be uniform and stable. Then, the electrometer was switched on. As no magnetic field had yet been applied to the electron tube, (the magnet was at this time far from the tube, $d \approx \infty, B \approx 0$ ), the tube should produce no output current, i.e. $B \approx 0, I \approx 0$. Actually, $I$ was not exactly zero at that time. There was a background current caused chiefly by the very small difference in temperature within the closed measuring circuit. We neglect the influence of the earth magnetic field. Of course, the weaker the background current, the better it was for our experiment.

We then applied a weak positive magnetic field to the tube, and denoted it by $B_{1}$. For example, $d=70 \mathrm{~cm}$, and $B_{t}=0.2$ gauss. The compass placed on the top of the copper box demonstrated the direction of the magnetic field, which should be adjusted parallel to the axis of the tube. We observed that the tube now put out a weak but stable current.

The magnetic induction intensity of the field was then increased in steps by reducing the distance $d$ between the tube and the magnet. During each step, we let the magnet remain stationary for a period of several minutes (so as to exclude disturbance of Faraday's electromagnetic induction), and we found that the output current remained stable in the period. From the beginning when $B_{\uparrow} \approx 0$ and $I \approx 0$, as $B_{\uparrow}$ increased in steps, the output current $I$ also increased in steps until it reached a maximum. After that, the output current decreased as the magnetic field was further increased. This drop down of the output current accorded with our expectation: as the magnetic field became stronger and stronger, the radii of thermal electrons became smaller and smaller, resulting in an increasing proportion of electrons that were no longer able to cross the gap to fly from $\mathrm{A}$ to $\mathrm{B}$, causing the output current to progressively reduce.

The magnet was now returned to the position $d=70 \mathrm{~cm}$, and rotated through $180^{\circ}$. The direction of the magnetic field in the copper shielding box consequently reversed. The magnetic field was now negative and denoted by $B_{\downarrow}$. As we expected, the direction of the output current also reversed. We then again reduced the distance $d$ in steps to increase the magnetic field $B$. The output current first increased, then decreased after reaching a maximum. The situation was similar in pattern to that with a positive magnetic field.

Further experiment showed that, in each step, provided the magnetic field remained stable (i.e., the magnet kept stationary), the output current $I$ would remain stable, with periods of stability possible for as long as several minutes, several hours, and even several days.

We call the output current Maxwell's current and denote it by I. In general, the output Maxwell's current for a given FX tube depends on two factors, the temperature $T$ and the magnetic induction intensity $B$.

$$
I=I(B, T) .
$$

Tables 2, 3 and 4 list the data from three tests at three different temperatures $10^{\circ} \mathrm{C}, 22^{\circ} \mathrm{C}$ and $32^{\circ} \mathrm{C}$. 
The corresponding $I \sim B$ graphs are shown in Figs. 8 (a), (b) and (c).

\begin{tabular}{|c|c|c|c|c|c|c|c|c|c|c|c|}
\hline$d(\mathrm{~cm})$ & $\infty$ & 60 & 50 & 45 & 40 & 37.5 & 35 & 30 & 25 & 20 & 15 \\
\hline$B($ gauss $)$ & 0 & 0.6 & 1.0 & 1.4 & 2.0 & 2.3 & 2.7 & 4.2 & 7.0 & 13 & 25 \\
\hline$I(f A)(B \uparrow)$ & 4.1 & 9 & 17 & 25 & 34 & 39 & 36 & 26 & 17 & 8 & 2.7 \\
\hline$I(f A)(B \downarrow)$ & 4.1 & 13 & 17 & 20 & 24 & 27 & 19 & 15 & 14 & 13 & 12 \\
\hline
\end{tabular}

Table $2 I \sim B$ relation of FX12-51 at $t=10^{\circ} \mathrm{C}$. Background current $I_{o}=4.1 \mathrm{fA}$.

\begin{tabular}{|c|c|c|c|c|c|c|c|c|c|c|}
\hline$d(\mathrm{~cm})$ & $\infty$ & 60 & 50 & 45 & 40 & 35 & 30 & 25 & 20 & 15 \\
\hline$B($ gauss $)$ & 0 & 0.6 & 1.0 & 1.4 & 2.0 & 2.7 & 4.2 & 7.0 & 13 & 25 \\
\hline$I(f A)(B \uparrow)$ & 3.0 & 45 & 85 & 117 & 165 & 182 & 152 & 127 & 104 & 94 \\
\hline$I(f A)(B \downarrow)$ & 3.0 & 53 & 72 & 78 & 59 & 43 & 26 & 22 & 20 & 17 \\
\hline
\end{tabular}

Table $3 I \sim B$ relation of FX12-51 at $t=22^{\circ} \mathrm{C}$. Background current $I_{o}=3.0 \mathrm{fA}$.

\begin{tabular}{|c|c|c|c|c|c|c|c|c|c|c|}
\hline$d(\mathrm{~cm})$ & $\infty$ & 60 & 50 & 45 & 40 & 35 & 30 & 25 & 20 & 15 \\
\hline$B($ gauss $)$ & 0 & 0.6 & 1.0 & 1.4 & 2.0 & 2.7 & 4.2 & 7.0 & 13 & 25 \\
\hline$I(f A)(B \uparrow)$ & 7.7 & 290 & 560 & 1360 & 1530 & 1650 & 1270 & 790 & 440 & 250 \\
\hline$I(f A)(B \downarrow)$ & 7.7 & 520 & 670 & 690 & 670 & 270 & 130 & 122 & 117 & 115 \\
\hline
\end{tabular}

Table $4 I \sim B$ relation of FX12-51at $t=32^{\circ} \mathrm{C}$. Background current $I_{o}=7.7 \mathrm{fA}$.

From $t=10^{\circ} \mathrm{C}$ to $t=33^{\circ} \mathrm{C}$, the temperature rose only $23^{\circ} \mathrm{C}$, nevertheless, the output current rose from 40fA to 1600fA, 40 times! This can be explained by Richardson's formula, thermal electron emission rises very rapidly as the temperature rises,

$$
J=A T^{2} e^{-\frac{W}{k T}} .
$$

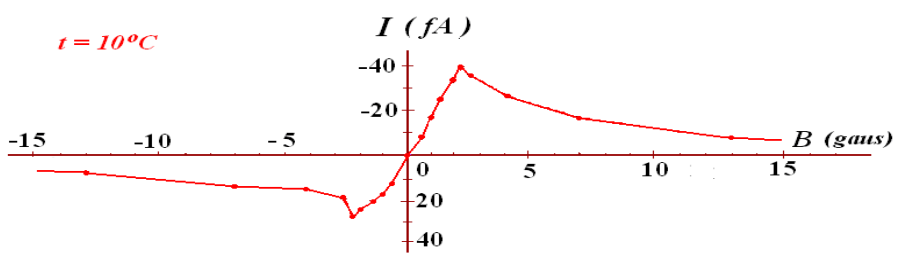

(a)

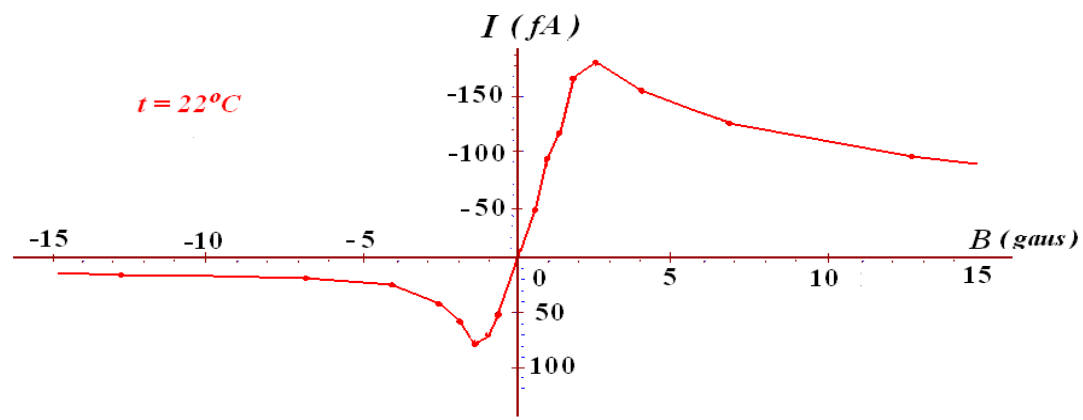

(b) 


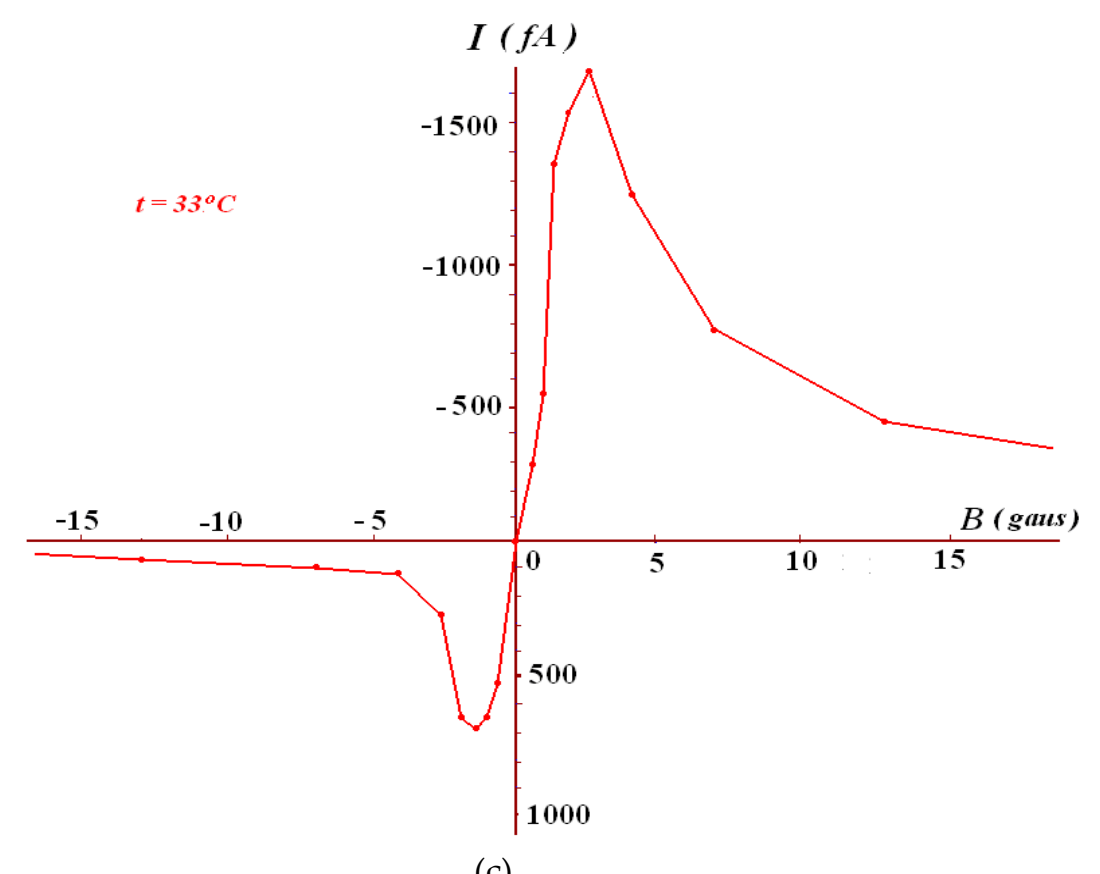

Fig 8 The $I \sim B$ curves of electron tube FX12-51 at three different temperatures.

The Keithley 6514 electrometer may also be used to measure the output voltage produced by the electron tube when a magnetic field is applied. The voltage here is simply the open-circuit voltage of the tube, or the electric motive force of the tube. This output voltage chiefly depends on the average kinetic energy of the thermal electrons of the emitters. Nevertheless, we noted that the leakage current between the two emitters might also affect the value of the output voltage.

The following is the output voltage we measured from electron tube FX12-51 in a test at a room temperature of $T=25^{\circ} \mathrm{C}(298 \mathrm{~K})$ :

Background voltage $B \approx 0, V_{o}=-5.6 \mathrm{mV}$.

The maxima of the output voltages were also relatively stable, each measured four times and list as follows

$$
\begin{array}{llrrr}
B \uparrow \approx 3.5 \text { gauss } & V=-20 & -21 & -20 & -21 \mathrm{mV}, \\
B \downarrow \approx 3.5 \text { gauss } & V=16 & 18 & 16 & 17 \mathrm{mV}
\end{array}
$$

According to Boltzmann's law of equi-partition of energy, the average kinetic energy of thermal electrons at $25^{\circ} \mathrm{C}(298 \mathrm{~K})$ is

$$
\bar{\varepsilon}=\frac{3}{2} k T=\frac{3}{2} \times 1.38 \times 10^{-23} \times 298 \mathrm{~J}=0.0385 \mathrm{eV}=0.0385 \mathrm{emV}
$$

In $38.5 \mathrm{em} V$, the factor $38.5 \mathrm{mV}$ is of the same order with the output voltage we measured in our experiment (about $20 \mathrm{mV}$ ). Therefore, we see, the output voltages were surely resulted from the conversion of part of the kinetic energy of the thermal electrons.

Both the output current and output voltage from our experiment were very weak, however, they were no doubt direct current and DC voltage, both being macroscopic ones. A large number of such Ag-O-Cs pairs could be connected in parallel to increase the output current, and connected in series to increase the output voltage, so as to build up a considerably greater electric power output.

\section{CONCLUSIONS}

In the above experiment, the heat extracted by the electron tube FX12-51 from the ambient air was converted completely into electric energy without producing any other effect. The process proves that the second law of thermodynamics is not universally valid, just as the two giants of physics, Maxwell and Planck, had predicted many years ago ${ }^{[3][4][5]}$.

The authors maintain: in ordinary thermodynamic processes, just as Clausius and Kelvin correctly pointed out, entropy always increases, never decreases. Nevertheless, in some specific or extraordinary thermodynamic processes, such as the process in our present experiment, entropy does decrease. 


\section{REFERENCES}

[1] Xinyong Fu, An Approach to Realize Maxwell's Hypothesis, Energy Conversion and Management, Vol.22 pp1-3 (1982).

[2] Xinyong Fu and Zitao Fu, Realization of Maxwell's Hypothesis, arxiv.org/physics/0311104V3

[3] James Clerk Maxwell, Theory of Heat, P.328, (1871)

[4] Max Planck, Forlesungen Uber Thermodynamik (Erste auflage,1897; Siebente auflage,(1922),§116 $\& \S 136$.

English Version: Treatise On Thermodynamics, Dover Publications Inc.(1926),§116 \&§136.

[5] W. Ehrenberg, Maxwell's Demon, Scientific American, pp.103-110 (1967)

[6] A. H. Sommer, PHOTOEMISSIVE MATERIALS, Preparation, Properties, and Use, John Wiley \& Sons (1968), Section 10.7.1, Chapter 10.

[7] John E. Davey, Thermionic and Semiconducting Properties of ( $\mathrm{Ag}$ ) - $\mathrm{Cs}_{2} \mathrm{O}_{3}, \mathrm{Ag}, \mathrm{Cs}$, Journal of Applied Physics, Volume 28, Number 9, p.1031 (1957)

(C) 2016 by the authors; licensee Preprints, Basel, Switzerland. This article is an open access article distributed under the terms and conditions of the Creative Commons by Attribution (CC-BY) license (http://creativecommons.org/licenses/by/4.0/). 